Draft VERSiOn June 21, 2021

Typeset using LATEX default style in AASTeX63

\title{
A Quasi-periodic Oscillation in the $\gamma$-ray Emission from the Non-blazar Active Galactic Nucleus PKS 0521-36
}

\author{
Haiyun Zhang, ${ }^{1}$ Dahai Yan, ${ }^{2}$ Pengfei Zhang, ${ }^{1}$ Shenbang Yang, ${ }^{1}$ And Li Zhang ${ }^{1}$ \\ ${ }^{1}$ Department of Astronomy, Key Laboratory of Astroparticle Physics of Yunnan Province, Yunnan University, \\ Kunming 650091, China \\ ${ }^{2}$ Key Laboratory for the Structure and Evolution of Celestial Objects, Yunnan Observatory, Chinese Academy of Sciences, \\ Kunming 650011, China
}

\begin{abstract}
Long-term $\gamma$-ray variability of a non-blazar Active Galactic Nucleus (AGN) PKS 0521-36 is investigated by using Fermi-LAT pass 8 data covering from 2008 August to 2021 March. The results show that the histogram of the $\gamma$-ray fluxes follows a log-normal distribution. Interestingly, in the analysis of 5.8-year (from MJD 56317 to 58447) LAT data between two outbursts (occurring during 2012 October and 2019 May respectively), a quasi-periodic oscillation (QPO) with a period of $\sim 1.1$ years $(\sim 5 \sigma$ of significance) is found in the Lomb-Scargle Periodogram (LSP), the Weighted Wavelet Z-transform (WWZ) and the REDFIT results. This quasi-periodic signal also appears in the results of Gaussian process modeling the light curve. Therefore, the robustness of the QPO is examined by four different methods. This is the first $\gamma$-ray QPO found in a mildly beamed jet. Our results imply that the $\gamma$-ray outbursts play an important role in the formation of the $\gamma$-ray QPO.
\end{abstract}

Keywords: Active galactic nuclei (16), Gamma-rays (637), Time series analysis (1916), Period search (1955), Jets (870)

\section{INTRODUCTION}

It is generally accepted that Active Galactic Nuclei (AGNs) existing in the center of a small fraction of galaxies are powerful emitters for the entire galaxies (e.g., Padovani et al. 2017). Usually, an AGN is powered by a central super massive black hole $(\mathrm{SMBH})$ and emits variable emission covering from radio to high-energy gamma-ray bands (e.g., Barkov et al. 2010; Madejski \& Sikora 2016). Non-thermal GeV-TeV $\gamma$-rays from AGNs are produced in their powerful relativistic jets.

The largest class of AGNs detected by Fermi Large Area Telescope (Fermi-LAT) is of blazar type (Ajello et al. 2020). A typical blazar has relativistic jet oriented at very small angle $\left(\theta<5^{\circ}\right)$ to the line of our sight (Ackermann et al. 2015), and therefore its jet emission is strongly Doppler-boosted (Urry \& Padovani 1995). Rapid flares on timescale of a few minutes in blazars have been observed at $\mathrm{GeV} \gamma$-ray energies (e.g., Ackermann et al. 2016; Meyer et al. 2019) and TeV energies (e.g., Aleksić et al. 2011). This kind of rapid flare suggests an extremely compact $\gamma$-ray emission region which likely locates in the broad-line region (BLR) of flat spectrum radio quasars (FSRQs). However, no signs of $\gamma$-ray absorption by BLR photons in $\gamma$-ray spectrum suggests that $\gamma$-ray emission region should be outside of the BLR (e.g., Costamante et al. 2018). To reconcile the observations of fast variability and simultaneous energy spectrum, new models are proposed (see Böttcher 2019; Blandford et al. 2019, for recent reviews). Moreover, timing analysis, such as the exploration of periodicity, would provide important information about the nature of these sources. Quasi-periodic variabilities of blazars in $\gamma$-ray energies have been investigated, and possible quasi-periodic variabilities in more than 20 blazars have been reported based on analyses of Fermi-LAT data (e.g., Sandrinelli et al. 2014, 2016, 2017; Ackermann et al. 2015; Prokhorov \& Moraghan 2017; Zhang et al. 2017a,c,b; Zhou et al. 2018; Bhatta 2019; Peñil et al. 2020; Zhang et al. 2020). However, the cause for the $\gamma$-ray quasi-periodic variabilities remains controversial.

Corresponding author: Dahai Yan

yandahai@ynao.ac.cn

Corresponding author: Li Zhang

lizhang@ynu.edu.cn 
Yan et al. (2018) investigated the relation between $\gamma$-ray variability amplitude and spectral index for PG 1553+113, and suggested that the $\gamma$-ray quasi-periodic variability of PG 1553+113 (Ackermann et al. 2015) could be caused by a periodic modulation in Doppler factor. Several possible explanations have been proposed for the $\gamma$-ray quasi-periodic variabilities in blazars, for instance, presence of a binary system of SMBHs (Valtonen et al. 2016; Tavani et al. 2018), modulation in the accretion flow feeding the jet (Gracia et al. 2003), and a periodic lighthouse effect (Camenzind \& Krockenberger 1992).

Seventy non-blazar AGNs are included in the Fourth Catalog of AGNs detected by Fermi-LAT (4LAC; Ajello et al. 2020). Among them, there are 41 radio galaxies (RGs), 9 narrow-line seyfert 1 galaxies (NLSy1s), 2 steep-spectrum radio quasars (SSRQs), 5 compact steep spectrum radio sources (CSSs), 1 Seyfert galaxy, and 11 other AGNs. 6 NLSy1s, 7 RGs, 3 CSSs, 1 SSRQ, and 3 other AGNs are found to be variable (Ajello et al. 2020). The jet properties of NLSy1 are similar to those of FSRQs, but with lower powers (e.g., D'Ammando et al. 2013, 2015a, 2016; Zhang et al. 2013; Paliya et al. 2019). $\gamma$-ray flares on a daily timescale are found in several NLSy1s (D'Ammando 2019). RGs are misaligned AGNs, and therefore the jet Doppler boosting effect is not strong. Intraday variability is found in the Fermi-LAT data of the RG NGC 1275 (Sahakyan et al. 2018; Tanada et al. 2018). All of the non-blazar AGNs are not bright enough to have good sampling in their long-term $\gamma$-ray light curves, except for NGC 1275 and PKS 0521-36. The $\gamma$-ray variability and spectral evolution of NGC 1275 have been studied in detail (e.g., Baghmanyan et al. 2017; Sahakyan et al. 2018; Tanada et al. 2018). Here, we focus on the $\gamma$-ray properties of PKS 0521-36. A combination of $\gamma$-ray properties of blazars and non-blazar AGNs can provide insightful information on AGN jet physics.

PKS 0521-36 is a peculiar non-blazar AGN with broad emission lines in the optical and ultraviolet bands and the steep radio spectrum (Scarpa et al. 1995; Keel 1986). D'Ammando et al. (2015b) raised a possibility that PKS 0521-36 is an intermediate source between broad-line radio galaxy and SSRQ. It was previously classified as an N galaxy, then a BL Lac object (D'Ammando et al. 2015b). The latest LAT source catalog (4FGL; Abdollahi et al. 2020) suggests that the existing data cannot determine a clear classification of this source. Very Long Baseline Array (VLBA) observations reveal that the radio structure of its jet is similar to misaligned AGN (D'Ammando et al. 2015b). Recently, Angioni et al. (2019) confirmed that the jet of PKS 0521-36 is not highly beamed.

The jet structure of PKS 0521-36 has been studied widely at frequencies from optical to X-rays (e.g., Scarpa et al. 1999; Falomo 1994; Keel 1986). Because of its prominent radio, optical, and X-ray jet (Danziger et al. 1979; Macchetto et al. 1991; Birkinshaw et al. 2002; Liuzzo et al. 2017), PKS 0521-36 is considered to be one of the most remarkable extragalactic objects. At $\gamma$-ray energies, a strong flare on variability timescale of 12 -hour was detected by Fermi-LAT from PKS 0521-36 in 2010 June (D'Ammando et al. 2015b). Recently, a $\gamma$-ray flare on timescale of $\sim 6$-hour in 2012 October was reported by Angioni et al. (2019).

A study on the long-term variability behavior of PKS 0521-36 in $\gamma$-ray energies is lack. The characteristics of $\gamma$-ray variability are helpful to understand the nature of this source. In this work, we perform a full time-domain analysis on the latest Fermi-LAT data of PKS 0521-36. The paper is organized as follows. The basic introduction of Fermi-LAT and results of searching for periodicity by different methods are presented in Section 2. Our discussion of the results is given in Section 3. Finally, a summary is presented in Section 4.

\section{DATA ANALYSIS AND RESULTS}

\subsection{Fermi-LAT Likelihood Analysis}

Fermi-LAT is a pair conversion $\gamma$-ray detector with wide field of view $(\sim 2.4 \mathrm{sr})$, large effective area $\left(>8000 \mathrm{~cm}^{2}\right.$ at $\sim 1 \mathrm{GeV}$ ), and broad-energy covering from $\sim 20 \mathrm{MeV}$ to $>300 \mathrm{GeV}$. Thanks to its all-sky monitoring capabilities, one can get long-coverage and uniform observations of $\gamma$-ray sources. A detailed description of LAT can be found in Atwood et al. (2009).

The data used here were collected during 12.6 years of Fermi-LAT operation from 2008 August 4 to 2021 March 29 [Modified Julian Day (MJD): 54682 - 59302] in the 0.1-500 GeV energy range. The analysis of data follows the standard criteria for the point-source analysis ${ }^{1}$. We employ the Science Tools package of version v11r05p3 available from Fermi Science Support Center ${ }^{2}$ (FSSC), and use the Pass 8 LAT database in which we choose a region of interest (ROI) of $15^{\circ}$ centered at PKS 0521-36. Two background templates, Galactic and extragalactic diffuse emissions, are modeled with the file gll_iem_v07.fits and iso_P8R3_SOURCE_ V2_v1.txt, respectively. The instrumental response

\footnotetext{
${ }^{1}$ http://fermi.gsfc.nasa.gov/ssc/data/analysis/documentation/Pass8_usage.html
}

2 https://fermi.gsfc.nasa.gov/ssc/data/analysis/software 
PKS0521-36

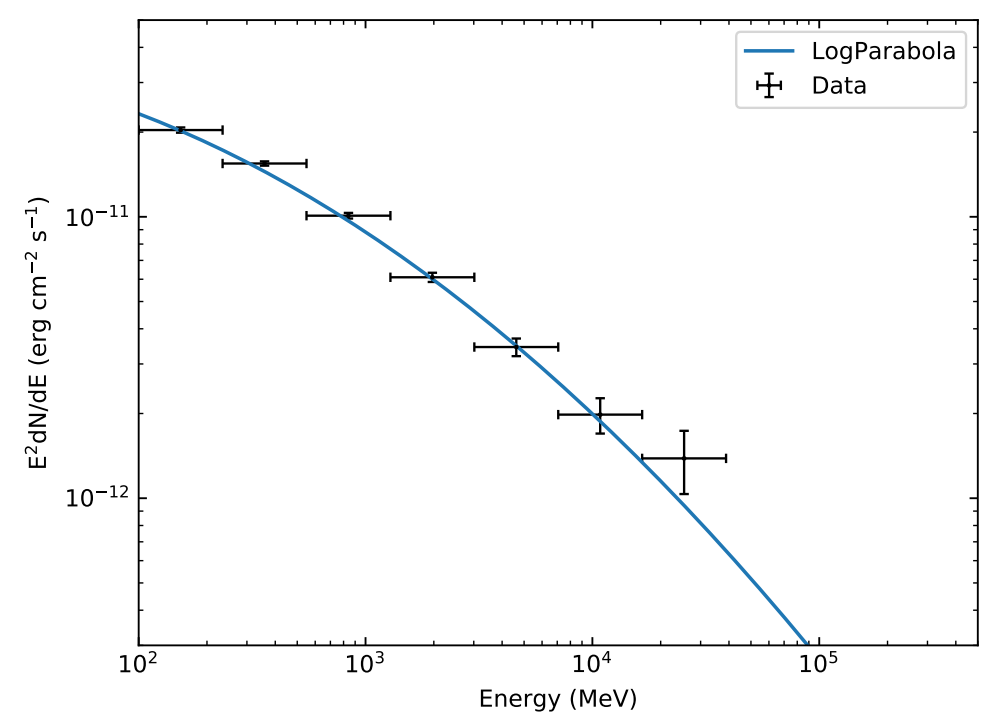

Figure 1. Average SED of PKS 0521-36 from MJD 54682 to 59302 . The best-fitting LP model is plotted as blue solid line, and the best-fitting parameters are given in the text.

function "P8R3_SOURCE_V2" is adopted in data processing. In order to get events with high probability of being photons, we only keep the SOURCE class events through setting "evclass $=128$, evtype $=3$ " in the Fermi tool gtselect. In addition, the events with zenith angle $>90^{\circ}$ are filtered out to avoid contaminating from Earth's limb. Good time intervals (GTIs) are selected by using the criterion of "(DATAQUAL >0)\&\&(LATCONFIG ==1)" in the Fermi tool gtmktime. Above settings ensure that we adopt high-quality observations.

\section{2. $\gamma$-ray SED and Light Curves}

In the process of analyzing the entire data, an initial binned maximum likelihood analysis (Mattox et al. 1996) is performed. The spectral parameters and the normalization factors of all sources lying within $5^{\circ}$ of PKS 0521-36 as well as the normalization factors of the sources located beyond $5^{\circ}$ but within $10^{\circ}$ from our target are set to be free, while the sources from ROI but beyond $10^{\circ}$ have their parameters fixed to the values in Fermi-LAT fourth source catalog (Abdollahi et al. 2020). In the iterative fittings, the best-fitting results are obtained, and are saved to be a new model file which is the basis of constructing light curves and spectrum energy distribution (SED). The spectral model of LogParabola (LP) form is used here, which is

$$
\frac{d N}{d E}=N_{0}\left(\frac{E}{E_{\mathrm{b}}}\right)^{-\left[\alpha+\beta \log \left(E / E_{\mathrm{b}}\right)\right]}
$$

The global fitting results in an integrated average flux of $(1.06 \pm 0.02) \times 10^{-7}$ photons $\mathrm{cm}^{-2} \mathrm{~s}^{-1}$ and a total Test Statistics (TS) value of 18447.2. The TS is defined as $2 \log \left(L / L_{0}\right)$, where $L$ is the maximum likelihood of the model with a point source at the target position, and $L_{0}$ is the maximum likelihood without the source (Mattox et al. 1996). The best-fitting spectral parameters are the spectral slope $\alpha=2.43 \pm 0.02$ and the curvature parameter $\beta=0.05 \pm 0.01$. The average SED of PKS 0521-36 in the whole time period is shown in Figure 1.

The unbinned maximum likelihood optimization is used to produce $\gamma$-ray light curve of PKS 0521-36. In each time bin, the new model file mentioned above is employed, but the spectral parameters of all sources are freezed. The time bins having $T S<25$ and the number of the model-predicted photon $N_{\text {pred }}<10$ are excluded . This ensures that the robust detections of the source are adopted in the analysis (Kapanadze et al. 2020; Raiteri et al. 2013). A 30-day binning light curve is produced. The light curve shown in Figure 2 reveals flux variability over the whole observational period. Three high flaring activities appear in the light curve. The first is in 2010 June and, has been reported by D'Ammando et al. (2015b) who also reported the contemporaneous increases of activities observed in optical, UV and 


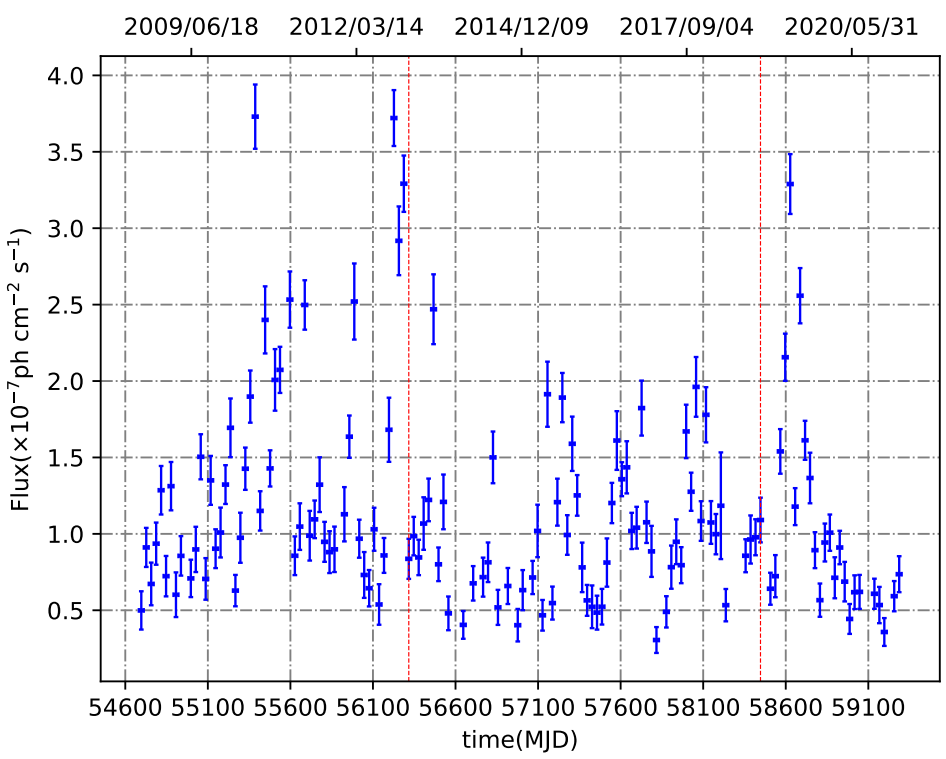

Figure 2. LAT light curve of PKS 0521-36 from MJD 54697 to 59287 in the energy range of $0.1 \mathrm{GeV}-500 \mathrm{GeV}$ with 30 days time bin. Two vertical dashed lines mark the time range of MJD 56317-58447, during which periodic modulation is analyzed (see Section 2.4).

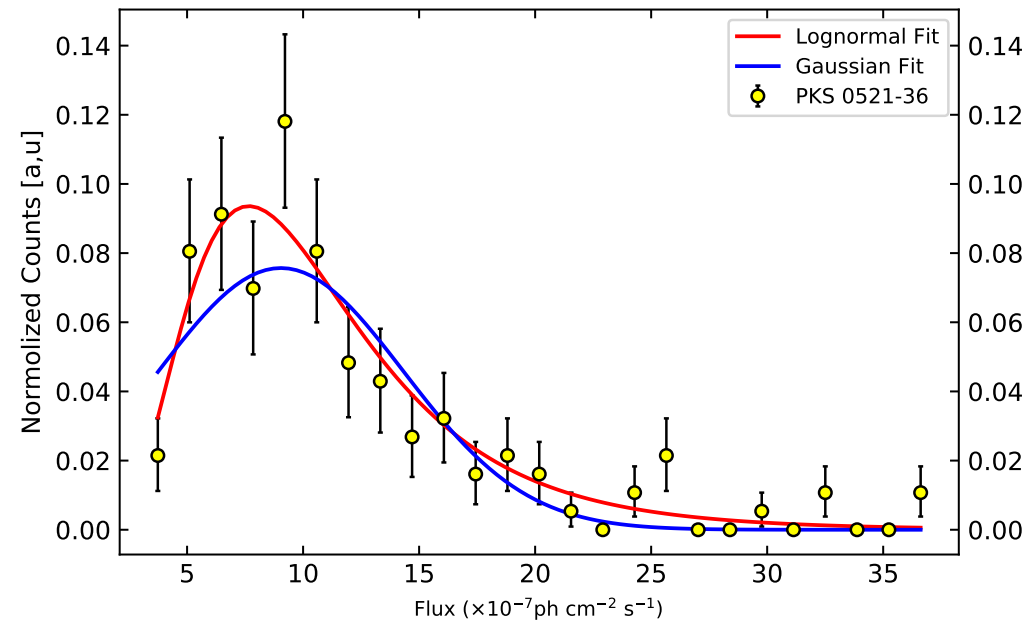

Figure 3. Normalized histogram of $\gamma$-ray photon fluxes fitted by the log-normal (red solid line) and Gaussian (blue solid line) function.

X-ray bands. The second flaring occurred in 2012 October has been studied by Angioni et al. (2019), and the latest flaring activity occurred in 2019 May.

\subsection{Flux Distribution}

One important property of a time series is its probability density function (PDF). One can explore the mechanism that drives variability by analyzing the flux distribution of a light curve (e.g., Rieger 2019). PDF can be estimated by fitting a histogram of observed data. The distribution of the $\gamma$-ray photon fluxes of PKS 0521-36 is presented in a 
Table 1. Best-fitting parameters for the log-normal and Gaussian distribution.

\begin{tabular}{|c|c|c|c|c|c|c|}
\hline \multirow[t]{2}{*}{ Time bin } & \multicolumn{3}{|c|}{ Log-normal } & \multicolumn{3}{|c|}{ Gaussian } \\
\hline & Mean* & $\sigma^{*}$ & $\chi_{\mathrm{red}}^{2}$ & Mean* & $\sigma^{*}$ & $\chi_{\text {red }}^{2}$ \\
\hline $30-d$ & $2.28 \pm 0.04$ & $0.49 \pm 0.04$ & 0.92 & $9.04 \pm 0.75$ & $5.27 \pm 0.71$ & 1.60 \\
\hline
\end{tabular}

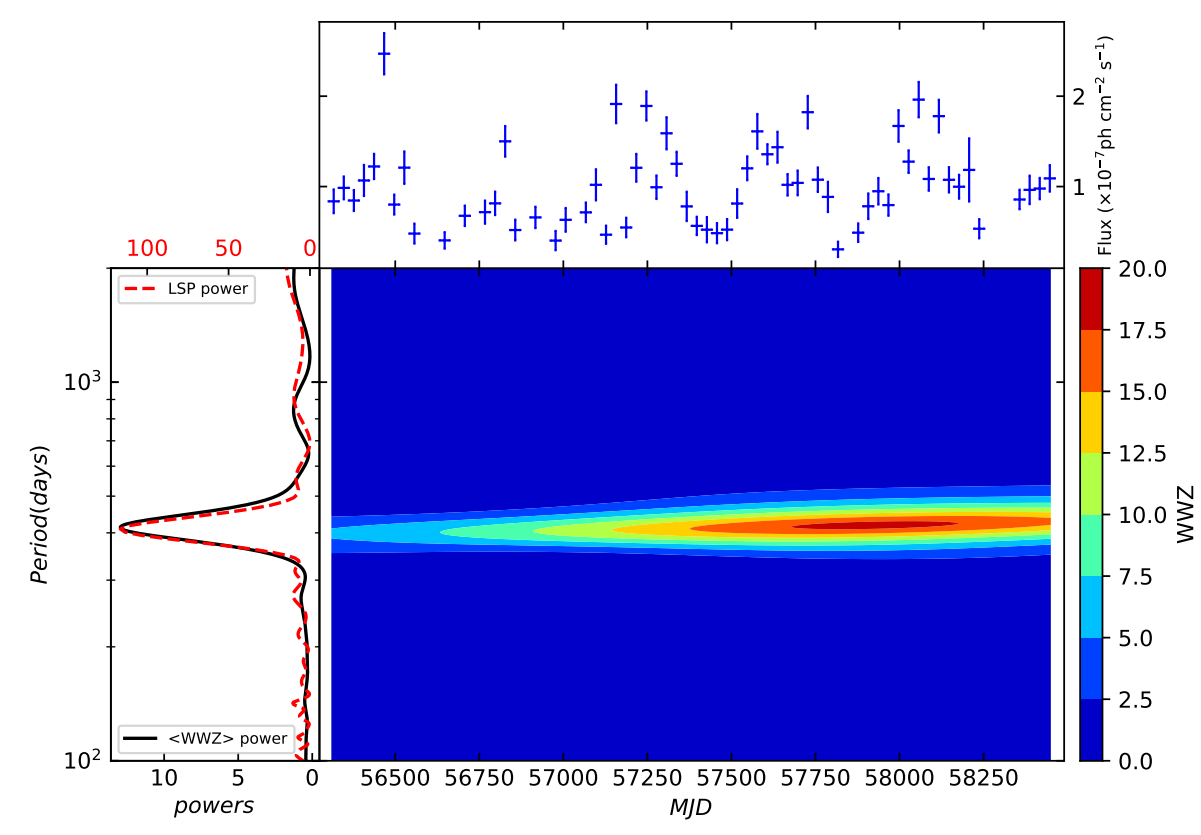

Figure 4. Upper panel: $\gamma$-ray light curve from MJD 56317 to 58447 with 30-day binning. Right lower panel: two dimensional contour map of the WWZ power spectrum of the $\gamma$-ray light curve. Left lower panel: LSP (red dashed line) and average WWZ (black solid line) powers of the light curve.

histogram with 25 bins, and is fitted by log-normal and Gaussian functions in the form of

$$
\begin{gathered}
L(\phi \mid \mu, \sigma)=\frac{1}{\sqrt{2 \pi} \sigma \phi} \exp \left[-\frac{(\log (\phi)-\mu)^{2}}{2 \sigma^{2}}\right], \\
G(\phi \mid \mu, \sigma)=\frac{1}{\sqrt{2 \pi} \sigma} \exp \left[-\frac{(\phi-\mu)^{2}}{2 \sigma^{2}}\right],
\end{gathered}
$$

respectively. The parameter $\sigma$ is the standard deviation and the parameter $\mu$ is the mean of the distribution. The flux histogram and best-fitting results are shown in Figure 3. The errors of data points are estimated by the method given by Gehrels (1986). The best-fitting parameters as well as the reduced $\chi^{2}$ obtained from the fittings with Gaussian and log-normal models are listed in table 1. It is found that the log-normal model is preferred over the Gaussian model.

\subsection{Searching for Periodicity}

The Lomb-Scargle periodogram (LSP; Lomb 1976; Scargle 1982) and the Weighted Wavelet Z-transform (WWZ; Foster 1996) are two of the most employed and best known methods for detection of periodicity in time-series in 


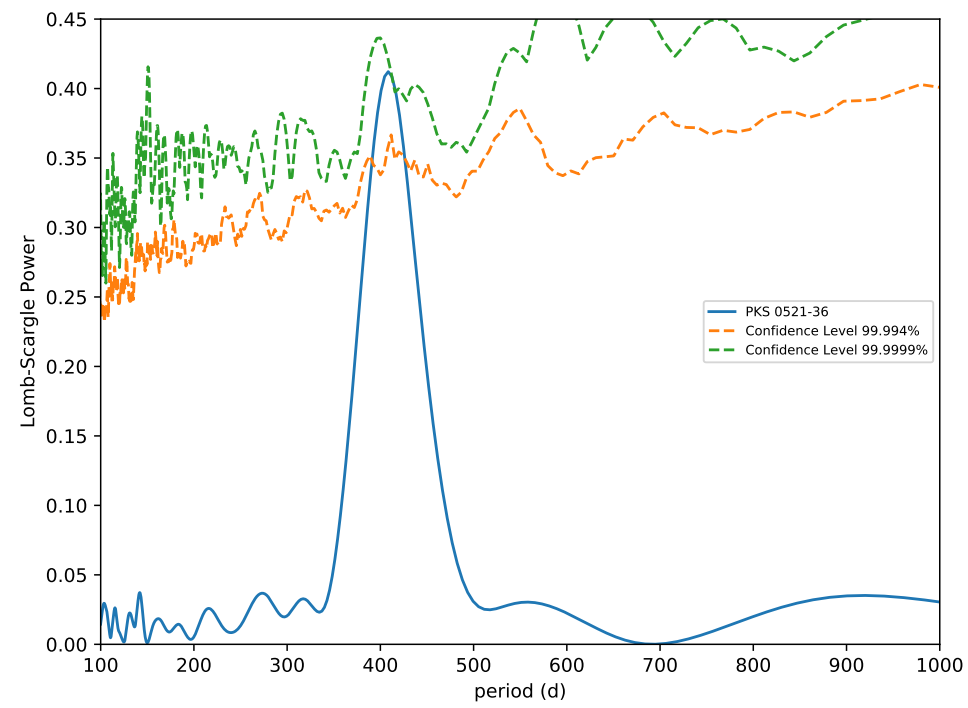

Figure 5. LSP power calculated from the light curve during MJD 56317-58447 (blue solid line), and the $4 \sigma$ (orange dashed line), $5 \sigma$ (green dashed line) significance curves calculated based on the simulation of $10^{5}$ light curves with the method given by Emmanoulopoulos et al. (2013).

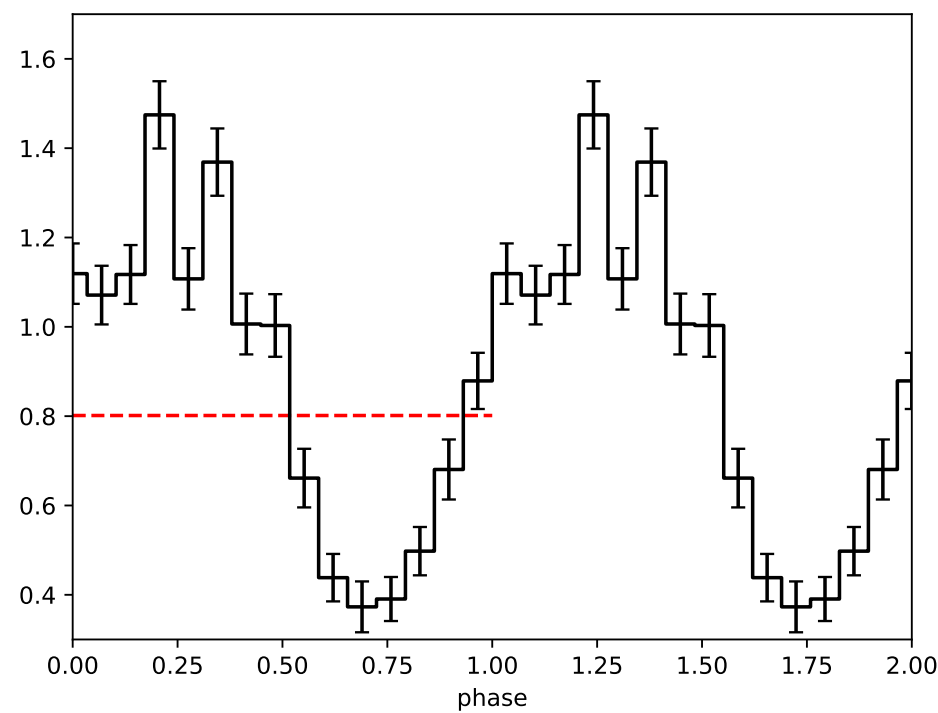

Figure 6. Folded light curve constructed from binned likelihood analysis of the data from MJD 56317-58447 above 100 MeV with a period of 411 days. The red dashed line is the constant fitting to the folded light curve in one phase. For clarity, we show two period cycles.

astronomy. The LSP and WWZ methods are then applied to the light curve of PKS 0521-36 to search for periodicity. A clear periodic modulation appears from MJD 56317 to 58447 (Figure 4). A significant peak at 400 days appears in both LSP and WWZ powers, which indicates a quasi-periodic oscillation (QPO). The period is estimated by fitting the power peak with a Gaussian function. It is $411 \pm 33$ days given by LSP and $417 \pm 39$ days given by WWZ. The bootstrap approximation method is used to calculate the false alarm probability (FAP), which is a robust way to estimate FAP 


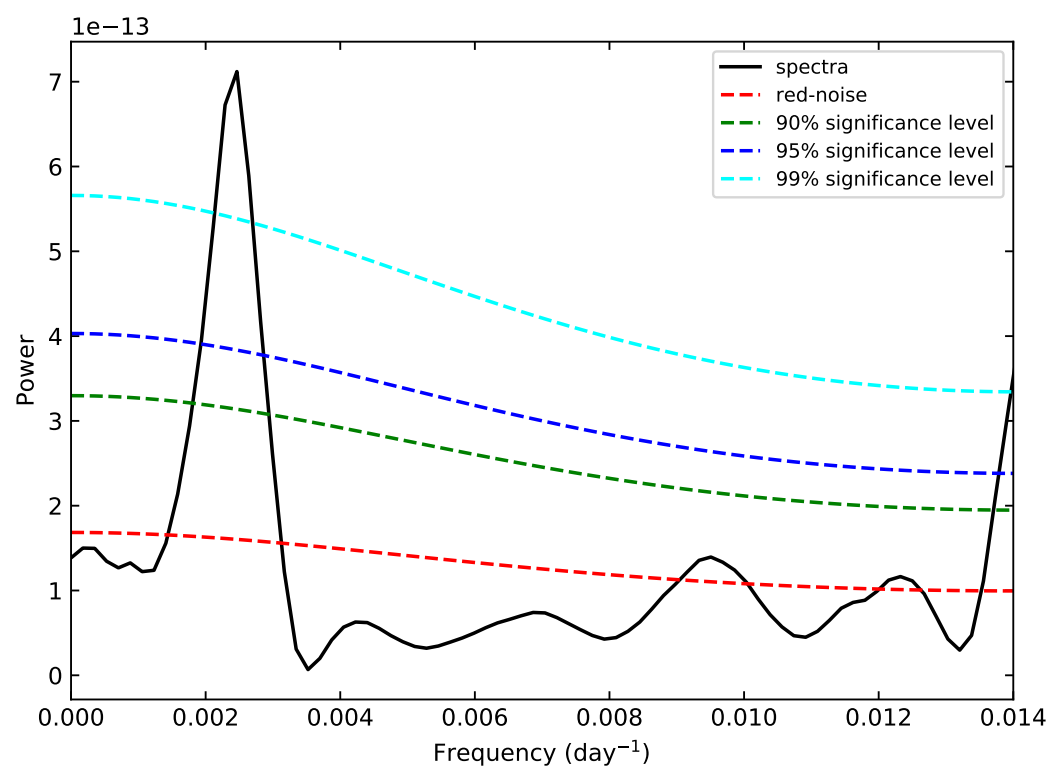

Figure 7. Results of the periodicity analysis by REDFIT. The black solid line is bias-corrected power spectrum. Dashed curves starting from the bottom are the theoretical red-noise spectrum, 90\%, 95\% and 99\% significance levels, respectively.

(e.g., Prokhorov \& Moraghan 2017; Peñil et al. 2020). The FAP is 0.0002 in the LSP power, corresponding to the $99.98 \%$ significance level for the signal.

The algorithm given by Emmanoulopoulos et al. (2013) is also used to evaluate the significance of this QPO signal. The properties of observed variation, power spectral density (PSD) and PDF, are well applied in this method. The PSD model of the form $P(f)=A f^{-\alpha}$ is used, where $\alpha$ is the power-law index. The PDF is in the form of a log-normal distribution. The $4 \sigma$ and $5 \sigma$ significance curves are obtained by simulating the 30 -day binning light curve $10^{5}$ times, and are shown in Figure 5. The significance of the QPO signal is $\approx 5 \sigma$.

Further evidence for this QPO is presented in the folded light curve constructed by performing the likelihood analysis method with a 411-day period. Phase zero is set at MJD 56317. The variation with the phase is clear (see Figure 6). The fitting to the folded light curve in one phase with a constant results in $\chi_{\text {red }}^{2}=449.26 / 14$, suggesting a significant variability in the folded light curve.

An additional method, REDFIT (Schulz \& Mudelsee 2002), is also employed for the periodicity detection. This method estimates the red-noise spectrum by fitting the data with a first-order autoregressive (AR1) process. It can precisely evaluate the significance of the peaks in the PSD against the red-noise background. We use the program REDFIT $3.8 \mathrm{e}^{3}$. The bias of Fourier transform for unevenly spaced data is also removed in the program. Two conditions should be satisfied when the program is used for data: (1) the data points of light curve should not be too clustered; and (2) the noise background of light curve can be approximated by an AR1 process. Obviously, our data meets the first condition. Moreover, the result of the non-parametric runs test for our data is rtest $=15$ (inside the $98 \%$ acceptance region $[9,22]$ ), which indicates that the noise background spectrum is consistent with an AR1 model (Schulz \& Mudelsee 2002). Therefore, REDFIT can be used to detect periodicity in our data. We set $n_{50}=2$ and select a hanning window to reduce spectral leakage (Schulz \& Stattegger 1997). The results show that there is a peak with more than $99 \%$ significance level, and the corresponding periodicity is $440.9 \pm 78.6$ days (Figure 7 ). This period is consistent with the LSP and the WWZ results. Note that the REDFIT method only provides a maximum of significance of $99 \%$.

\subsection{Gaussian Process Modeling the $\gamma$-ray Light Curve}

Besides LSP, WWZ and REDFIT, an alternative approach for the analysis of astronomical variability is the Gaussian process modeling light curves, which treats the variability in the time rather than frequency domain (e.g., Kelly et al. 2009, 2014; Li \& Wang 2018). The PSD can be constructed by using modeling results. One popular Gaussian process

\footnotetext{
3 https://www.marum.de/Prof.-Dr.-michael-schulz/Michael-Schulz-Software.html
} 

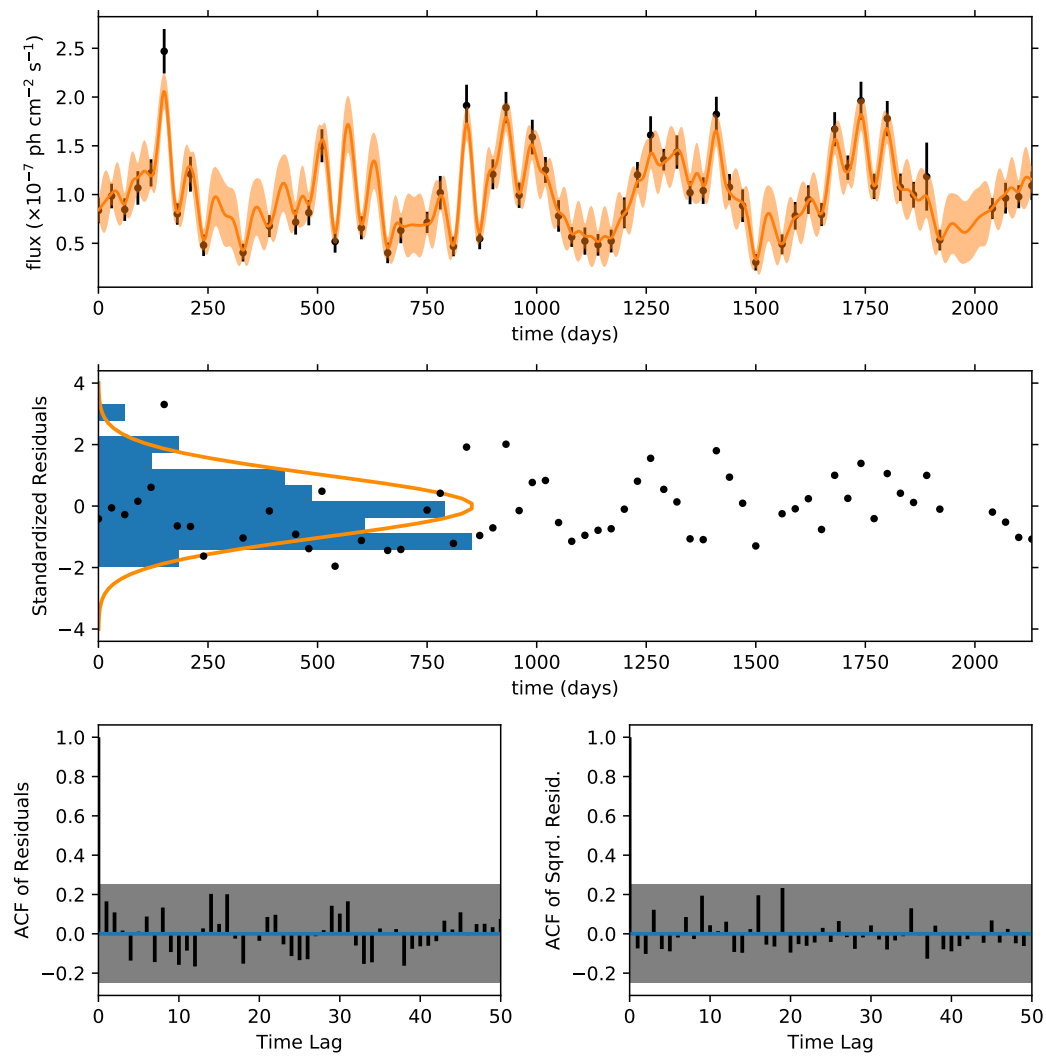

Figure 8. celerite modeling results for the 30-day binning $\gamma$-ray light curve of PKS 0521-36 from MJD 56317 to 58447; in the top plot, black points with errors are LAT data, and the bright orange line is the best fit to the data with the celerite model; black dots, blue histogram and orange line in the middle plot are standardized residuals of each bin, scaled standardized residuals distribution, and the expected normal distribution (scaled), respectively; the ACFs of residuals and squared residuals are given in the bottom plot where the gray region is the $95 \%$ confidence limit of the white noise.

model is the continuous time autoregressive moving average (CARMA) model developed by Kelly et al. (2014), which has been applied to $\gamma$-ray variability of blazars (e.g., Goyal et al. 2018; Ryan et al. 2019; Yang et al. 2021). Here a newly developed Gaussian process model celerite (Foreman-Mackey et al. 2017), is used to investigate the $\gamma$-ray variability of PKS 0521-36.

In this work, a stochastically driven damped simple harmonic oscillator (SHO) is used, and its differential equation is given by

$$
\left[\frac{d^{2}}{d t^{2}}+\frac{\omega_{0}}{Q} \frac{d}{d t}+\omega_{0}^{2}\right] y(t)=\epsilon(t)
$$

where $\omega_{0}, Q, \epsilon(t)$ are the frequency of the undamped oscillator, the quality factor of the oscillator, and a stochastic driving force (white noise here), respectively. The PSD in the case is

$$
S(\omega)=\sqrt{\frac{2}{\pi}} \frac{S_{0} \omega_{0}^{4}}{\left(\omega^{2}-\omega_{0}^{2}\right)^{2}+\omega^{2} \omega_{0}^{2} / Q^{2}},
$$

where $S_{0}$ is proportional to the power when $\omega=\omega_{0}$.

A specific model for a time series can be built up with a mixture of determined number of SHO terms. The Markov Chain Monte Carlo (MCMC) algorithm (Foreman-Mackey et al. 2013) is used to perform fitting to light curve. From MCMC sampling, we can evaluate values and uncertainties of model parameters. In detail, $5 \times 10^{4}$ samples are generated in our analysis, and the first $2 \times 10^{4}$ samples are taken as burn-in sampling.

Figure 8 shows the fitting results to the 30-day binning light curve of PKS 0521-36 from MJD 56317 to 58447 with the celerite model $(\mathrm{SHO} \times 2)$. For the modeling results, we use Kolmogorov-Smirnov $(\mathrm{KS})$ test to check the consistency between the standardized residuals and a normal distribution with mean zero and standard deviation of unity. The 

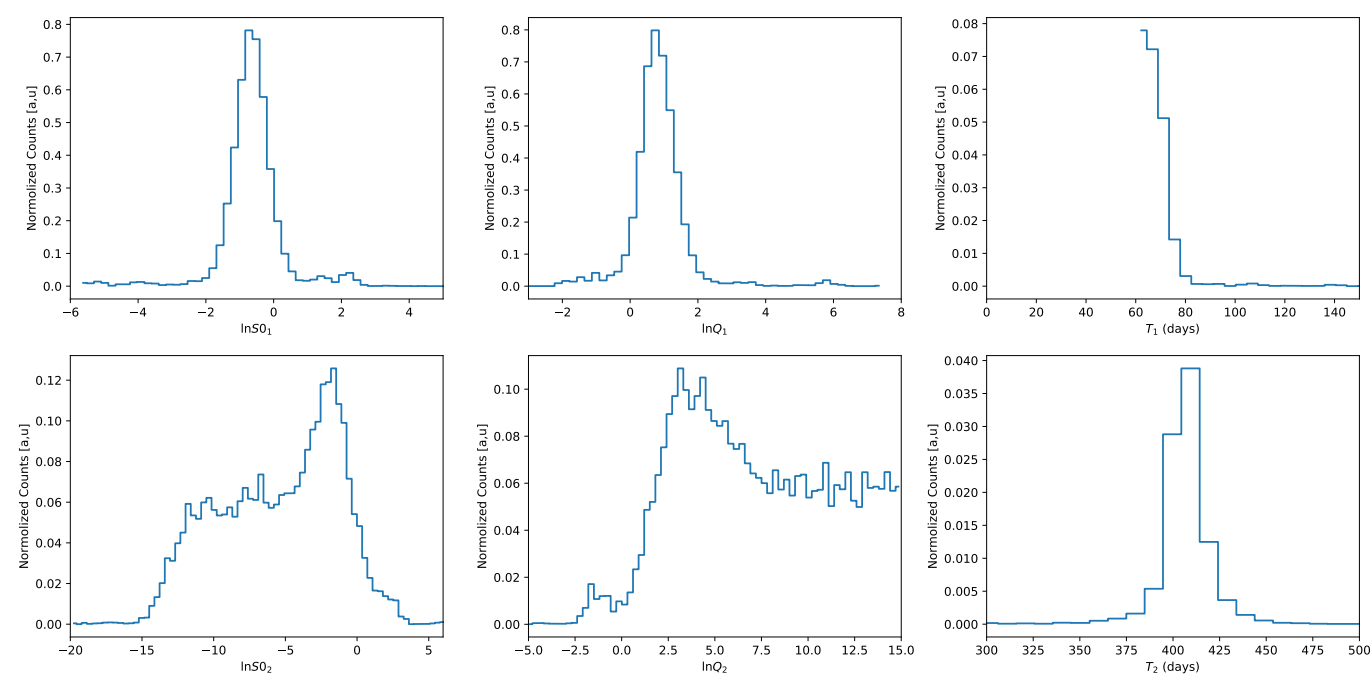

Figure 9. Posterior probability densities of parameters obtained from celerite modeling results.

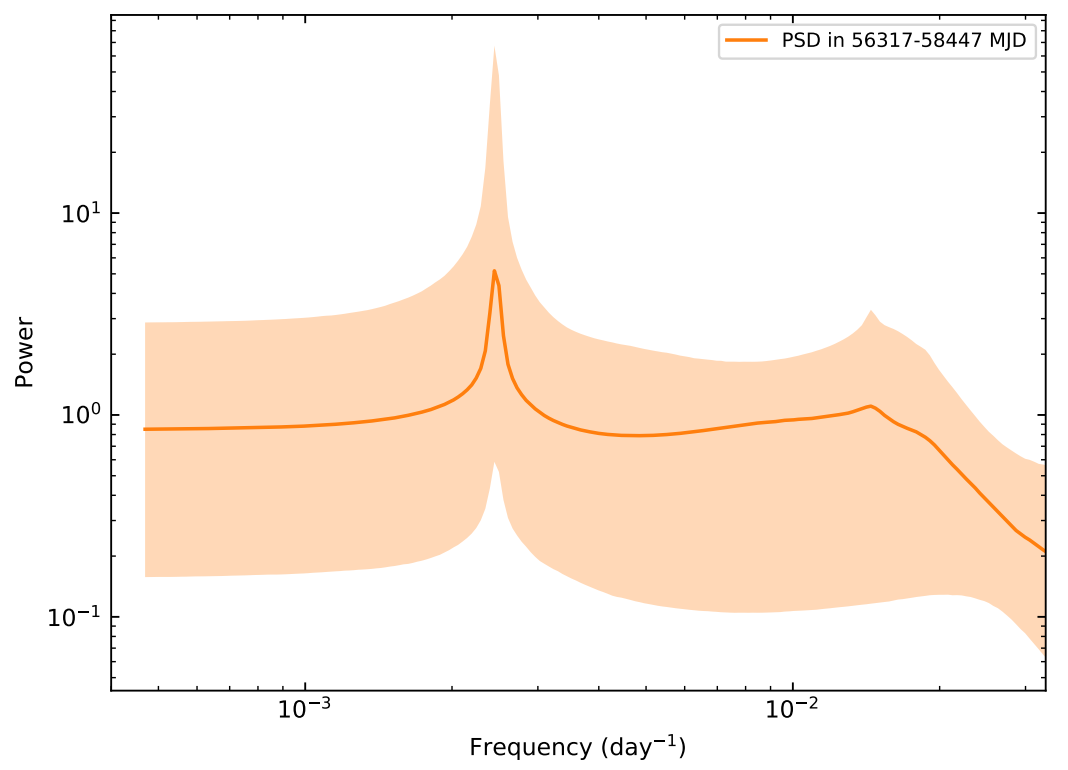

Figure 10. PSD constructed from the celerite modeling results for the light curve during MJD 56317-58447. Bright color region is the $68 \%$ confidence interval. A distinct peak appears at $\sim 400$ days.

$\mathrm{p}$-values of the test is $\mathrm{p}=0.23$, which means this consistency is credible (Yang et al. 2021). The auto-correlation functions (ACFs) of the residuals and squared residuals in Figure 8 are almost inside the $95 \%$ confidence limits, so that the correlation structures have been captured by the model. These results indicate that the $\gamma$-ray variability can be described well by the two-SHO model. The posterior probability densities for the model parameters are shown in Figure 9 and the value of these parameters are given in Table 2. A QPO feature with period of $\sim 400$ days appears in the PSD constructed from the modeling results (Figure 10).

There is an aperiodic component and a periodic component in the $\gamma$-ray variability during the time range of MJD 56317-58447. The period of the QPO is estimated as $406 \pm 10$ days which is consistent with the results given by LSP, WWZ and REDFIT. The corresponding quality factor of the oscillator is large (Table 2), suggesting that the QPO is significant (Foreman-Mackey et al. 2017). 
Table 2. Parameters for the celerite modeling results corresponding to Figure 9.

\begin{tabular}{ccccc}
\hline \hline Time & SHO No. & $\ln S_{0}$ & $\ln Q$ & $T$ \\
$(\mathrm{MJD})$ & & & $(5)$ \\
$(1)$ & $(2)$ & $(3)$ & $(4)$ & $66.5^{+5.18}$ \\
\hline $56317-58447$ & 1 & $-0.65_{-0.53}^{+0.54}$ & $0.80_{-0.5}^{+0.55}$ & $405.93_{-10.32}^{+9.68}$ \\
\hline
\end{tabular}

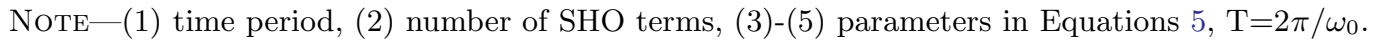

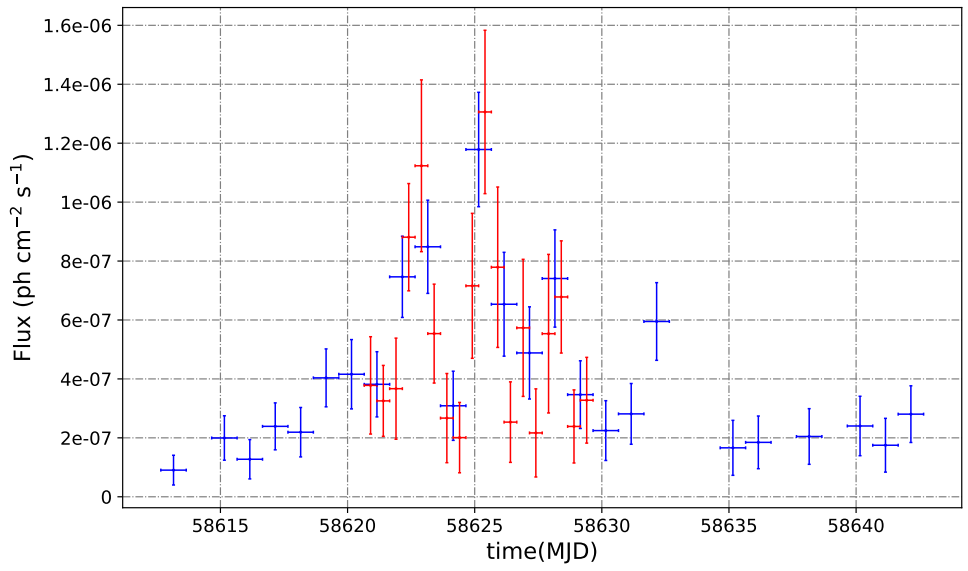

Figure 11. One-day binning light curve (blue points) and 12-hour binning light curve (red points) for the bright flare during 2019 May 9-June 8 (MJD 58612-58642).

\subsection{Short Timescale Variability}

Intraday $\gamma$-ray variability of PKS 0521-36 has been found in the LAT data (D'Ammando et al. 2015b; Angioni et al. 2019). A recent bright $\gamma$-ray flare occurred in 2019 May 9-June 8 (MJD 58612-58642) has not been investigated. In our work, intraday variability during this flare is observed in the one-day and half-day binning light curves (Figure 11). Here we use unbinned maximum likelihood method to construct the light curves, and select time bins having TS $\geqslant 4$. This flare is not bright enough to use the selection criteria of TS $\geqslant 25$ for such a short time bin.

In order to determine variability timescale, the 12-hour binning light curve is fitted by using the following function (Abdo et al. 2010)

$$
F(t)=F_{\mathrm{c}}+F_{0}\left(e^{\frac{t_{0}-t}{T_{\mathrm{r}}}}+e^{\frac{t-t_{0}}{T_{\mathrm{d}}}}\right)^{-1}
$$

where $F_{\mathrm{c}}, F_{0}, t_{0}, T_{\mathrm{r}}$ and $T_{\mathrm{d}}$ are a constant flux, peak flux, peak time, and rise and decay times, respectively. This function can be used to describe one-peak flare. The light curve with multi-peaks is considered as a whole, and is fitted by using the model comprising several components of Equation 6 . The best-fitting results are presented in Figure 12, and the parameters are given in Table 3. The residuals are fitted by the constant of zero successfully with $\chi_{\text {red }}^{2}=0.58$, suggesting that our fitting to the light curve is acceptable. It is found $T_{\mathrm{r}} \sim T_{\mathrm{d}} \sim 7$ hours. This is the third short timescale $\gamma$-ray flare in PKS 0521-36. However, the loose data selection criteria of TS $\geqslant 4$ decreases the significance of the derived timescale.

The SEDs during 2019 May 9 - June 8 (MJD 58612-58642) and 2012 October 12 - November 11 (MJD 56212-56242) are also produced. The spectral parameters are listed in Table 4 , together with the parameters for the average SED 

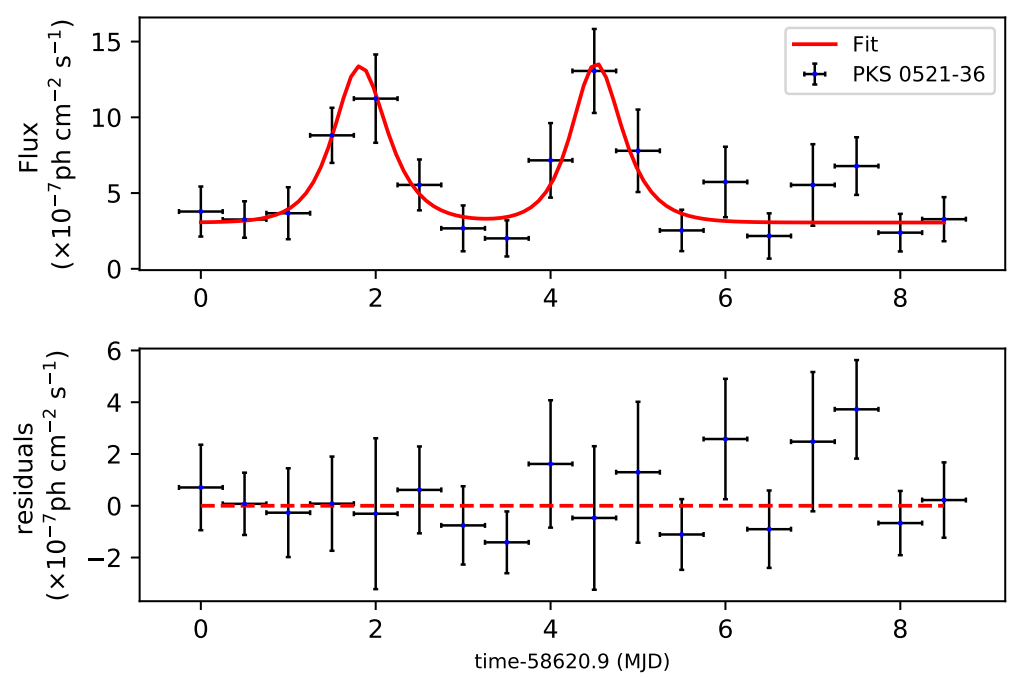

Figure 12. Best-fitting results for the 12-hour binning light curve showed in Figure 11 with Equation 6, and the distribution of the residuals obtained in the fitting (bottom panel) which is fitted by a constant (red dashed line).

Table 3. Best-fitting parameters for the 12-hour light curve in Figure 12.

\begin{tabular}{cccccccc}
\hline \hline $\begin{array}{c}\text { Time } \\
\text { (MJD) }\end{array}$ & Peak No. & $F_{\mathrm{c}}$ & $F_{0}$ & $t_{0}$ & $T_{\mathrm{r}}$ & $T_{\mathrm{d}}$ & $\chi_{\text {red }}^{2}$ \\
$(1)$ & & & & & $($ days $)$ & (days) & (days) \\
& $(2)$ & $(3)$ & $(4)$ & $(5)$ & $(6)$ & $(7)$ & $(8)$ \\
\hline \multirow{2}{*}{$58620.9-58629.4$} & $\mathrm{p} 1$ & $3.06 \pm 0.54$ & $20.63 \pm 8.81$ & $1.80 \pm 0.31$ & $0.25 \pm 0.27$ & $0.29 \pm 0.18$ & 0.90 \\
& $\mathrm{p} 2$ & & $20.95 \pm 5.55$ & $4.5^{*}$ & $0.24 \pm 0.11$ & $0.28 \pm 0.12$ & \\
\hline
\end{tabular}

Note-(1) time period, (2) peak number, (3)-(7) parameters in Equations 6 , (8) the reduced $\chi^{2},(3)(4)$ in unit of $10^{-7}$ photons $\mathrm{cm}^{-2} \mathrm{~s}^{-1}$. The value with $*$ represents that it is fixed in fitting.

Table 4. Best-fitting spectral parameters in four periods.

\begin{tabular}{cccccc}
\hline \hline $\begin{array}{c}\text { Time period } \\
(\mathrm{MJD})\end{array}$ & $\begin{array}{c}\text { Time period } \\
(\mathrm{UT})\end{array}$ & $\alpha$ & $\beta$ & TS & Flux \\
\hline $54682-59287$ & $2008-08-04 / 2021-03-29$ & $2.43 \pm 0.02$ & $0.05 \pm 0.01$ & 18447.2 & $1.06 \pm 0.02$ \\
$56212-56242$ & $2012-10-12 / 2012-11-11$ & $2.41 \pm 0.05$ & -- & 1363.98 & $3.70 \pm 0.21$ \\
$58612-58642$ & $2019-05-09 / 2019-06-08$ & $2.39 \pm 0.08$ & -- & 851.68 & $3.22 \pm 0.24$ \\
$56317-58447$ & $2013-01-25 / 2018-11-25$ & $2.46 \pm 0.03$ & $0.06 \pm 0.02$ & 6188.17 & $0.91 \pm 0.02$ \\
\hline
\end{tabular}

shown in Figure 1. It is found that during the two flares, the SEDs are the same, which can be described by a power-law form, different from the average LP SED.

\section{DISCUSSION}

Our results show that the PDF obtained from the $\gamma$-ray light curve of PKS 0521-36 from 2008 August 4 to 2021 March 29 is preferentially log-normally distributed. The log-normal distribution of flux is found in X-ray binaries (e.g., 
Uttley \& McHardy 2001), X-ray NLSy1s (e.g., Uttley et al. 2005), and blazars (e.g., Sinha et al. 2016; Shah et al. 2018; Ait Benkhali et al. 2020; Bhatta \& Dhital 2020). Similar as X-ray binaries, the log-normal distribution of $\gamma$-ray fluxes may indicate an influence of the accretion disk on the jet (Giebels \& Degrange 2009), i.e., the multiplicative behavior in an accretion disc impacting the jet. In this scenario, the variability timescale is dominated by the viscous timescale of the accretion disc (Rieger 2019). However, the tentative timescale of $\sim 7$ hours $\gamma$-ray variability found in PKS 0521-036 is unlikely achieved in this scenario (Czerny 2006). The cascade-related emission processes may also lead to a log-normal distribution of fluxes (Rieger 2019), for instance, the cascade emission in lepto-hadronic jet model (e.g., Mannheim 1993; Böttcher et al. 2013; Yan \& Zhang 2015). In this case, the observed GeV emission is produced by the cascade processes, which is feasible. However, it would require an extremely high jet power (e.g., Petropoulou et al. 2015; Cerruti 2020).

A simple and natural scenario is that the log-normal distribution of variability results from the emitting particles with the log-normal distribution (Sinha et al. 2018; Rieger 2019). This interpretation is associated with the fluctuations in the particle acceleration rate (Sinha et al. 2018). The average LP SED suggests an average LP electron distribution. Both the log-normal and LP electron distributions could be the result of stochastic acceleration (Tramacere et al. 2011).

Possible $\gamma$-ray QPOs have been reported in blazars (e.g., Ackermann et al. 2015; Prokhorov \& Moraghan 2017; Peñil et al. 2020; Sandrinelli et al. 2014, 2016, 2017; Zhang et al. 2017a,c; Zhou et al. 2018), although cautions have been raised on the significance of these QPOs (e.g., Covino et al. 2019, 2020; Ait Benkhali et al. 2020; Yang et al. 2021). It is well known that blazar jets are highly beamed. So far, no $\gamma$-ray QPO is reported in non-blazar AGNs.

Here, we report a $\gamma$-ray QPO with a significance of $\sim 5 \sigma$ in non-blazar AGN PKS 0521-36. The period is $\sim 1.1$ years. This QPO behavior only appears in the temporal range from MJD 56317 to 58447 which is between two outbursts (occurred in 2012 October and 2019 May respectively). This significant QPO is obtained by three different periodicity detection methods (LSP, WWZ and REDFIT). Evidence for this QPO is also found in the Gaussian process modeling the 30-day binning $\gamma$-ray light curve. It is confirmed that the jet of PKS 0521-36 is a mildly beamed (Leon et al. 2016; Angioni et al. 2019). Therefore, the QPO of PKS 0521-36 is the first $\gamma$-ray QPO found in a mildly beamed jet. The average SED during MJD 56317-58447 is described by a LP model (see Table 4) which is different from the power-law SEDs in the two outbursts.

The detection of a helicoidal motion in the optical jet of PKS 0521-36 was reported (Jiménez-Andrade et al. 2017), which may be associated with helical magnetic field or jet precession. A simultaneous VLBI core flux activity during the $\gamma$-ray flare in 2010-2011 was reported by Angioni et al. (2019). This suggests that the $\gamma$-ray emission region is located inside the radio core rather in the jet. $\gamma$-ray variability on timescale of $\sim 7$ hours is observed before and after the QPO. If the $\gamma$-ray photons produced in a single region, the helicoidal motion along the jet at pc scales (Jiménez-Andrade et al. 2017) could not lead to the $\gamma$-ray QPO of PKS 0521-36.

It seems that the outburst during 2012 October plays a key role in producing this QPO. It is not clear whether the outburst during 2019 May destroys this QPO or triggers another QPO. The data collected in future several years will answer this question.

\section{SUMMARY}

We have carried out a temporal analysis of the $\gamma$-ray emissions from an interesting non-blazar AGN PKS 0521-36 with the Fermi-LAT data from 2008 August 4 to 2021 March 29. Our main results are as follows.

(i) The PDF of the $\gamma$-ray fluxes is more consistent with a log-normal distribution rather than a Gaussian distribution. It may be due to the log-normal distribution of emitting electrons, which could be the result of stochastic acceleration. Moreover, the total average LP SED also can be produced by the stochastic acceleration.

(ii) Variability on timescale of $\sim 7$ hours is found during the outburst in 2019 May. It is the third short timescale $\gamma$-ray flare in PKS 0521-36. Such a variability indicates that the $\gamma$-ray emission region is compact. However, it should be noted that the timescale may be in low significance.

(iii) A QPO with the period of $\sim 1.1$ years is found at $\sim 5 \sigma$ confidence level in the time interval (MJD 56317-58447) between two outbursts. Four different methods are used to examine the robustness of the QPO. All the methods give significant evidence for this QPO. This is the first $\gamma$-ray QPO found in a mildly beamed jet. The SED during the QPO interval is different from that during the two $\gamma$-ray outbursts in 2012 October and 2019 May. It is argued that the two $\gamma$-ray outbursts play a key role in the formation of this $\gamma$-ray QPO. The $\gamma$-ray variability during the QPO interval can be well modeled by two SHO components, an aperiodic component and a periodic component. 


\section{ACKNOWLEDGMENTS}

We thank the anonymous reviewer for constructive suggestions. We acknowledge financial support from National Key R\&D Program of China under grant No. 2018YFA0404204, the National Science Foundation of China 11803081, and the joint foundation of Department of Science and Technology of Yunnan Province and Yunnan University [2018FY001(-003)]. The work of D. H. Yan is also supported by the CAS Youth Innovation Promotion Association and Basic research Program of Yunnan Province (202001AW070013).

Facility: Fermi(LAT)

Software: Fermitools-conda, celerite (Foreman-Mackey et al. 2017), emcee (Foreman-Mackey et al. 2013), REDFIT (Schulz \& Mudelsee 2002), NumPy (Harris et al. 2020), Matplotlib (Hunter 2007), Astropy (Astropy Collaboration et al. 2013, 2018), SciPy (Virtanen et al. 2020), DELCgen (Connolly 2016).

\section{REFERENCES}

Abdo, A. A., Ackermann, M., Ajello, M., et al. 2010, ApJ, 722, 520, doi: 10.1088/0004-637X/722/1/520

Abdollahi, S., Acero, F., Ackermann, M., et al. 2020, ApJS, 247, 33, doi: 10.3847/1538-4365/ab6bcb

Ackermann, M., Ajello, M., Atwood, W. B., et al. 2015, ApJ, 810, 14, doi: 10.1088/0004-637X/810/1/14

Ackermann, M., Anantua, R., Asano, K., et al. 2016, ApJL, 824, L20, doi: 10.3847/2041-8205/824/2/L20

Ait Benkhali, F., Hofmann, W., Rieger, F. M., \& Chakraborty, N. 2020, A\&A, 634, A120, doi: 10.1051/0004-6361/201935117

Ajello, M., Angioni, R., Axelsson, M., et al. 2020, ApJ, 892, 105, doi: 10.3847/1538-4357/ab791e

Aleksić, J., Antonelli, L. A., Antoranz, P., et al. 2011, ApJL, 730, L8, doi: 10.1088/2041-8205/730/1/L8

Angioni, R., Ros, E., Kadler, M., et al. 2019, A\&A, 627, A148, doi: 10.1051/0004-6361/201935697

Astropy Collaboration, Robitaille, T. P., Tollerud, E. J., et al. 2013, A\&A, 558, A33, doi: 10.1051/0004-6361/201322068

Astropy Collaboration, Price-Whelan, A. M., Sipőcz, B. M., et al. 2018, AJ, 156, 123, doi: 10.3847/1538-3881/aabc4f

Atwood, W. B., Abdo, A. A., Ackermann, M., et al. 2009, ApJ, 697, 1071, doi: 10.1088/0004-637X/697/2/1071

Baghmanyan, V., Gasparyan, S., \& Sahakyan, N. 2017, ApJ, 848, 111, doi: 10.3847/1538-4357/aa8c7b

Barkov, M. V., Aharonian, F. A., \& Bosch-Ramon, V. 2010, ApJ, 724, 1517, doi: 10.1088/0004-637X/724/2/1517

Bhatta, G. 2019, MNRAS, 487, 3990, doi: $10.1093 / \mathrm{mnras} / \mathrm{stz} 1482$

Bhatta, G., \& Dhital, N. 2020, ApJ, 891, 120, doi: $10.3847 / 1538-4357 / \mathrm{ab} 7455$

Birkinshaw, M., Worrall, D. M., \& Hardcastle, M. J. 2002, MNRAS, 335, 142, doi: 10.1046/j.1365-8711.2002.05615.x

Blandford, R., Meier, D., \& Readhead, A. 2019, ARA\&A, 57, 467, doi: 10.1146/annurev-astro-081817-051948
Böttcher, M. 2019, Galaxies, 7, 20, doi: 10.3390/galaxies7010020

Böttcher, M., Reimer, A., Sweeney, K., \& Prakash, A. 2013, ApJ, 768, 54, doi: 10.1088/0004-637X/768/1/54

Camenzind, M., \& Krockenberger, M. 1992, A\&A, 255, 59

Cerruti, M. 2020, Galaxies, 8, 72, doi: 10.3390/galaxies 8040072

Connolly, S. D. 2016, DELightcurveSimulation: Light curve simulation code. http://ascl.net/1602.012

Costamante, L., Cutini, S., Tosti, G., Antolini, E., \& Tramacere, A. 2018, MNRAS, 477, 4749, doi: $10.1093 / \mathrm{mnras} / \mathrm{sty} 887$

Covino, S., Landoni, M., Sandrinelli, A., \& Treves, A. 2020, ApJ, 895, 122, doi: 10.3847/1538-4357/ab8bd4

Covino, S., Sandrinelli, A., \& Treves, A. 2019, MNRAS, 482, 1270, doi: 10.1093/mnras/sty2720

Czerny, B. 2006, in Astronomical Society of the Pacific Conference Series, Vol. 360, AGN Variability from X-Rays to Radio Waves, ed. C. M. Gaskell, I. M. McHardy, B. M. Peterson, \& S. G. Sergeev, 265

D'Ammando, F. 2019, Galaxies, 7, 87, doi: 10.3390/galaxies7040087

D'Ammando, F., Orienti, M., Finke, J., et al. 2013, MNRAS, 436, 191, doi: 10.1093/mnras/stt1560

—. 2015a, MNRAS, 446, 2456, doi: 10.1093/mnras/stu2251

D'Ammando, F., Orienti, M., Tavecchio, F., et al. 2015b, MNRAS, 450, 3975, doi: 10.1093/mnras/stv909

D'Ammando, F., Orienti, M., Finke, J., et al. 2016, MNRAS, 463, 4469, doi: 10.1093/mnras/stw2325

Danziger, I. J., Fosbury, R. A. E., Goss, W. M., \& Ekers, R. D. 1979, MNRAS, 188, 415, doi: $10.1093 /$ mnras $/ 188.2 .415$

Emmanoulopoulos, D., McHardy, I. M., \& Papadakis, I. E. 2013, MNRAS, 433, 907, doi: 10.1093/mnras/stt764

Falomo, R. 1994, The Messenger, 77, 49 
Foreman-Mackey, D., Agol, E., Ambikasaran, S., \& Angus, R. 2017, AJ, 154, 220, doi: 10.3847/1538-3881/aa9332

Foreman-Mackey, D., Hogg, D. W., Lang, D., \& Goodman, J. 2013, PASP, 125, 306, doi: 10.1086/670067

Foster, G. 1996, AJ, 112, 1709, doi: 10.1086/118137

Gehrels, N. 1986, ApJ, 303, 336, doi: 10.1086/164079

Giebels, B., \& Degrange, B. 2009, A\&A, 503, 797, doi: 10.1051/0004-6361/200912303

Goyal, A., Stawarz, Ł., Zola, S., et al. 2018, ApJ, 863, 175, doi: 10.3847/1538-4357/aad2de

Gracia, J., Peitz, J., Keller, C., \& Camenzind, M. 2003, MNRAS, 344, 468, doi: 10.1046/j.1365-8711.2003.06832.x

Harris, C. R., Millman, K. J., van der Walt, S. J., et al. 2020, Nature, 585, 357-362, doi: 10.1038/s41586-020-2649-2

Hunter, J. D. 2007, Computing in Science and Engineering, 9, 90, doi: 10.1109/MCSE.2007.55

Jiménez-Andrade, E. F., Chavushyan, V., León-Tavares, J., et al. 2017, MNRAS, 470, L107, doi: 10.1093/mnrasl/slx090

Kapanadze, B., Gurchumelia, A., Dorner, D., et al. 2020, ApJS, 247, 27, doi: 10.3847/1538-4365/ab6322

Keel, W. C. 1986, ApJ, 302, 296, doi: 10.1086/163991

Kelly, B. C., Bechtold, J., \& Siemiginowska, A. 2009, ApJ, 698, 895, doi: 10.1088/0004-637X/698/1/895

Kelly, B. C., Becker, A. C., Sobolewska, M., Siemiginowska, A., \& Uttley, P. 2014, ApJ, 788, 33, doi: 10.1088/0004-637X/788/1/33

Leon, S., Cortes, P. C., Guerard, M., et al. 2016, A\&A, 586, A70, doi: 10.1051/0004-6361/201527146

Li, Y.-R., \& Wang, J.-M. 2018, MNRAS, 476, L55, doi: $10.1093 / \mathrm{mnrasl} / \mathrm{sly} 028$

Liuzzo, E., Paladino, R., \& Galluzzi, V. 2017, in Submm/mm/cm QUESO Workshop 2017 (QUESO2017), 16, doi: 10.5281/zenodo.1038079

Lomb, N. R. 1976, Ap\&SS, 39, 447, doi: $10.1007 / \mathrm{BF} 00648343$

Macchetto, F., Albrecht, R., Barbieri, C., et al. 1991, ApJL, 369, L55, doi: 10.1086/185957

Madejski, G. G., \& Sikora, M. 2016, ARA\&A, 54, 725, doi: 10.1146/annurev-astro-081913-040044

Mannheim, K. 1993, A\&A, 269, 67. https://arxiv.org/abs/astro-ph/9302006

Mattox, J. R., Bertsch, D. L., Chiang, J., et al. 1996, ApJ, 461, 396, doi: 10.1086/177068

Meyer, M., Scargle, J. D., \& Blandford, R. D. 2019, ApJ, 877, 39, doi: 10.3847/1538-4357/ab1651

Padovani, P., Alexander, D. M., Assef, R. J., et al. 2017, A\&A Rv, 25, 2, doi: 10.1007/s00159-017-0102-9
Paliya, V. S., Parker, M. L., Jiang, J., et al. 2019, ApJ, 872, 169, doi: 10.3847/1538-4357/ab01ce

Peñil, P., Domínguez, A., Buson, S., et al. 2020, ApJ, 896, 134, doi: 10.3847/1538-4357/ab910d

Petropoulou, M., Dimitrakoudis, S., Padovani, P., Mastichiadis, A., \& Resconi, E. 2015, MNRAS, 448, 2412, doi: 10.1093/mnras/stv179

Prokhorov, D. A., \& Moraghan, A. 2017, MNRAS, 471, 3036, doi: 10.1093/mnras/stx1742

Raiteri, C. M., Villata, M., D'Ammando, F., et al. 2013, MNRAS, 436, 1530, doi: 10.1093/mnras/stt1672

Rieger, F. 2019, Galaxies, 7, 28, doi: 10.3390/galaxies7010028

Ryan, J. L., Siemiginowska, A., Sobolewska, M. A., \& Grindlay, J. 2019, ApJ, 885, 12, doi: 10.3847/1538-4357/ab426a

Sahakyan, N., Baghmanyan, V., \& Zargaryan, D. 2018, A\&A, 614, A6, doi: 10.1051/0004-6361/201732304

Sandrinelli, A., Covino, S., Dotti, M., \& Treves, A. 2016, AJ, 151, 54, doi: 10.3847/0004-6256/151/3/54

Sandrinelli, A., Covino, S., \& Treves, A. 2014, ApJL, 793, L1, doi: 10.1088/2041-8205/793/1/L1

Sandrinelli, A., Covino, S., Treves, A., et al. 2017, A\&A, 600, A132, doi: 10.1051/0004-6361/201630288

Scargle, J. D. 1982, ApJ, 263, 835, doi: 10.1086/160554

Scarpa, R., Falomo, R., \& Pian, E. 1995, A\&A, 303, 730

Scarpa, R., Urry, C. M., Falomo, R., \& Treves, A. 1999, ApJ, 526, 643, doi: 10.1086/308041

Schulz, M., \& Mudelsee, M. 2002, Computers and Geosciences, 28, 421, doi: 10.1016/S0098-3004(01)00044-9

Schulz, M., \& Stattegger, K. 1997, Computers and Geosciences, 23, 929, doi: 10.1016/S0098-3004(97)00087-3

Shah, Z., Mankuzhiyil, N., Sinha, A., et al. 2018, Research in Astronomy and Astrophysics, 18, 141, doi: 10.1088/1674-4527/18/11/141

Sinha, A., Khatoon, R., Misra, R., et al. 2018, MNRAS, 480, L116, doi: 10.1093/mnrasl/sly136

Sinha, A., Shukla, A., Saha, L., et al. 2016, A\&A, 591, A83, doi: 10.1051/0004-6361/201628152

Tanada, K., Kataoka, J., Arimoto, M., et al. 2018, ApJ, 860, 74, doi: 10.3847/1538-4357/aac26b

Tavani, M., Cavaliere, A., Munar-Adrover, P., \& Argan, A. 2018, ApJ, 854, 11, doi: 10.3847/1538-4357/aaa3f4

Tramacere, A., Massaro, E., \& Taylor, A. M. 2011, ApJ, 739, 66, doi: 10.1088/0004-637X/739/2/66

Urry, C. M., \& Padovani, P. 1995, PASP, 107, 803, doi: $10.1086 / 133630$

Uttley, P., \& McHardy, I. M. 2001, MNRAS, 323, L26, doi: 10.1046/j.1365-8711.2001.04496.x 
Uttley, P., McHardy, I. M., \& Vaughan, S. 2005, MNRAS, 359, 345, doi: 10.1111/j.1365-2966.2005.08886.x

Valtonen, M. J., Zola, S., Ciprini, S., et al. 2016, ApJL, 819, L37, doi: 10.3847/2041-8205/819/2/L37

Virtanen, P., Gommers, R., Oliphant, T. E., et al. 2020, Nature Methods, 17, 261, doi: 10.1038/s41592-019-0686-2

Yan, D., \& Zhang, L. 2015, MNRAS, 447, 2810, doi: $10.1093 / \mathrm{mnras} / \mathrm{stu} 2551$

Yan, D., Zhou, J., Zhang, P., Zhu, Q., \& Wang, J. 2018, ApJ, 867, 53, doi: 10.3847/1538-4357/aae48a

Yang, S., Yan, D., Zhang, P., Dai, B., \& Zhang, L. 2021, ApJ, 907, 105, doi: 10.3847/1538-4357/abcbff
Zhang, J., Liang, E.-W., Sun, X.-N., et al. 2013, ApJL, 774, L5, doi: 10.1088/2041-8205/774/1/L5

Zhang, P.-f., Yan, D.-h., Liao, N.-h., \& Wang, J.-c. 2017a, ApJ, 835, 260, doi: 10.3847/1538-4357/835/2/260

Zhang, P.-f., Yan, D.-h., Liao, N.-h., et al. 2017b, ApJ, 842, 10, doi: 10.3847/1538-4357/aa7465

Zhang, P.-F., Yan, D.-H., Zhou, J.-N., et al. 2017c, ApJ, 845, 82, doi: 10.3847/1538-4357/aa7ecd

Zhang, P.-f., Yan, D.-h., Zhou, J.-n., Wang, J.-c., \& Zhang, L. 2020, ApJ, 891, 163, doi: 10.3847/1538-4357/ab71fe

Zhou, J., Wang, Z., Chen, L., et al. 2018, Nature Communications, 9, 4599, doi: 10.1038/s41467-018-07103-2 